\title{
Radiographic features and prognosis of early- and late-onset non-small cell lung cancer immune checkpoint inhibitor- related pneumonitis
}

Aiben Huang ${ }^{1 \dagger}$, Yang $\mathrm{Xu}^{2+}$, Xuelei Zang ${ }^{3+}$, Chongchong $\mathrm{Wu}^{4}$, Jie Gao ${ }^{5+}$, Xiaoli Sun ${ }^{1}$, Mei Xie ${ }^{6 \dagger}$, Xidong $\mathrm{Ma}^{2}$, Hui Deng ${ }^{1}$, Jialin Song ${ }^{7}$, Fangping Ren ${ }^{1}$, Li Pang ${ }^{1}$, Jin Qian ${ }^{1}$, Zhaofeng Y ${ }^{8}$, Shiyu Wan ${ }^{8}$, Yuanyuan Chen ${ }^{8}$, Lei Pan ${ }^{1 *}$, Guanglei Zhuang ${ }^{*^{*}}$, Sanhong Liu ${ }^{10^{*}}$ and Xinying Xue ${ }^{*^{*}}$

\begin{abstract}
Background: Immunotherapy is becoming a standard of care for non-small cell lung cancer (NSCLC). Checkpoint inhibitor-associated pneumonia (CIP) is a rare and potentially life-threatening event that can occur at any time during tumor immunotherapy. However, there may be differences in the radiological patterns and prognosis of CIP during different periods. This study aimed to investigate the radiographic features and prognosis of early- and lateonset immune-related pneumonitis.

Methods: We retrospectively analyzed the clinical data of 677 NSCLC patients receiving immunotherapy to identify 32 patients with CIP, analyzed the clinical and radiographic data, and summarized the radiological features and prognosis of early- and late-onset CIP.

Results: CIP had an incidence of $4.7 \%$, a median onset time of 10 weeks, and a mortality of $28.1 \%$. Among these, CIP included 14 early-onset cases, where grade $\geq 3$ CIP accounted for $92.9 \%$, main radiographic pattern was organizing pneumonia (OP)-like pattern, and mortality was 50.0\%. We also identified 18 late-onset CIPs, where grade $\geq 3$ CIP accounted for $50.0 \%$, main radiographic pattern was nonspecific interstitial pneumonia (NSIP)-like pattern, and mortality was $11.1 \%$. The overall survival rate of the early-onset group was significantly lower than that of the late-onset group $(P<0.05)$.
\end{abstract}

\footnotetext{
*Correspondence: leipan2010@163.com; zhuangguanglei@163.com; liush@shutcm.edu.cn; xuexinying2988@bjsjth.cn

${ }^{+}$Aiben Huang, Yang Xu, Xuelei Zang, Chongchong Wu and Jie Gao contributed equally to this work.

${ }^{1}$ Department of Respiratory and Critical Care, Beijing Shijitan Hospital, Capital Medical University, Beijing, China

${ }^{9}$ Shanghai Key Laboratory of Gynecologic Oncology, Renji Hospital, School of

Medicine, Shanghai Jiao Tong University, Shanghai, China

${ }^{10}$ Institute of Interdisciplinary Integrative Medicine Research, Shanghai University of Traditional Chinese Medicine, Shanghai, China

Full list of author information is available at the end of the article
}

(c) The Author(s). 2021 Open Access This article is licensed under a Creative Commons Attribution 4.0 International License, which permits use, sharing, adaptation, distribution and reproduction in any medium or format, as long as you give appropriate credit to the original author(s) and the source, provide a link to the Creative Commons licence, and indicate if changes were made. The images or other third party material in this article are included in the article's Creative Commons. licence, unless indicated otherwise in a credit line to the material. If material is not included in the article's Creative Commons licence and your intended use is not permitted by statutory regulation or exceeds the permitted use, you will need to obtain permission directly from the copyright holder. To view a copy of this licence, visit http://creativecommons.org/licenses/by/4.0/ The Creative Commons Public Domain Dedication waiver (http://creativecommons.org/publicdomain/zero/1.0/) applies to the data made available in this article, unless otherwise stated in a credit line to the data. 
Conclusion: Early-onset CIP cases were higher in the Common Terminology Criteria for Adverse Events (CTCAE v5.0) grade and mainly presented with an OP-like radiographic pattern; whereas, late-onset CIP cases were lower in CTCAE grade and mainly presented with an NSIP-like radiographic pattern. Finally, the prognosis of the early-onset CIP group was poorer than that of the late-onset CIP group. We believe that this study will be helpful for clinicians for making early diagnosis and deciding treatment modalities for patients with CIP.

Keywords: Immunotherapy, NSCLC, Checkpoint inhibitor-associated pneumonia, Prognosis, Radiographic patterns

\section{Background}

In the last decade, immune checkpoint inhibitors (ICIs) have played an increasingly important role in the treatment of advanced non-small cell lung cancer (NSCLC), malignant melanoma, and other malignancies [1, 2]. However, with the continuously expanding indications of ICIs and their widespread application in clinical front-line treatment, many immune-related adverse reactions associated with ICIs have been observed, for instance, in the skin, pituitary gland, thyroid gland, liver, kidney, lung, and other organs; these reactions can even be life-threatening $[3,4]$.

Checkpoint inhibitor-associated pneumonia (CIP) is a rare fatal immune-related adverse event (irAE), which has an incidence rate of $2-5 \%$ [5]. Nishino et al. found that the incidence of CIP is higher in NSCLC than in melanoma [6]. Further, compared with patients with other malignant tumors, NSCLC patients had a higher mortality rate associated with ICI treatment $[7,8]$. CIP could occur at any time during tumor immunotherapy [9], and typically varies from 2 to 24 months [10]. Nakahama et al. [11], Erwin et al. [12], and Costa et al. [13] have found that CIP can occur within a few days after ICI application. However, the clinical manifestations of these patients were inconsistent, for instance, some patients developed respiratory failure quickly; whereas, other patients showed no obvious discomfort, even though their chest computed tomography (CT) showed an obvious exudation shadow. Erwin et al. found that patients with early CIP have severe clinical symptoms [12]. Furthermore, the radiographic pattern of CIP is complicated. In addition to the typical cryptogenic organizing pneumonia (COP)-like, nonspecific interstitial pneumonia (NSIP)-like, hypersensitivity pneumonia (HP)-like, and acute interstitial pneumonia (AIP)-like interstitial patterns, there are some uncommon radiographic patterns such as sarcoid-like granulomatosis [14] and pleural effusion [15]. However, there are no reports that evaluate the radiographic difference between early- and late-onset CIP. The aim of this study was to investigate the imaging patterns and prognosis of CIP in different stages.

\section{Methods}

\section{Subjects}

We retrospectively analyzed the clinical data of 677 NSCLC patients who received ICI treatment (anti- programmed death 1 /programmed death ligand 1) between January 2017 and September 2020 at one of two hospitals (the General Hospital of the people's Liberation Army and Affiliated Beijing Shijitan Hospital of Capital Medical University). A total of 32 patients with CIP were identified, and patients with infection or tumor progression were excluded. Further, patients who had received epidermal growth factor receptor-tyrosine kinase inhibitor (EGFR-TKI) were also excluded to rule out EGFRTKI-induced interstitial lung disease (ILD). Postoperative histopathology, CT-guided lung puncture biopsy, or tracheoscopic bronchial mucosa biopsy was used to identify the 677 patients with NSCLC. Clinical data of the patients included sex, age, smoking history, underlying diseases, allergy history, tumor location, pathological pattern, degrees of differentiation, clinical stages, clinical symptoms, treatment methods, and overall survival. All patients were followed up from the time of initiation of ICI therapy to death or last research phone call by a member of the research team. The study was approved by the ethics committee of the hospital.

\section{CIP, peritumoral infiltration (PTI), and criteria for CIP classification}

The diagnosis of CIP was determined by the treating oncologist (Mei Xie) and confirmed by a multidisciplinary irAE team consisting of a pulmonologist (Aiben Huang), radiologist (Xiaoli Sun) and a second oncologist (Chongchong $\mathrm{Wu}$ ). For identifying CIP patients, we considered patients who developed dyspnea or other respiratory symptoms (including cough, shortness of breath, etc.) after treatment with ICIs, along with the presence of new radiographic infiltration and lack of evidence of pulmonary infection or other alternative etiologies (tumor progression, radiation pneumonitis, diffuse alveolar hemorrhage, heart failure, etc.). Briefly, using a combination of clinical (history and examination, arterial blood gas analysis, and pulmonary function testing), radiographic (presence or absence of tumor progression and pattern of parenchymal infiltrates), and biologic parameters (white blood cell count, sputum and/or bronchoalveolar lavage cultures, respiratory viral cultures, and cytopathologic testing), we excluded alternative etiologies such as heart failure, infection, and tumor progression. Improvement was defined as decrease in oxygen 


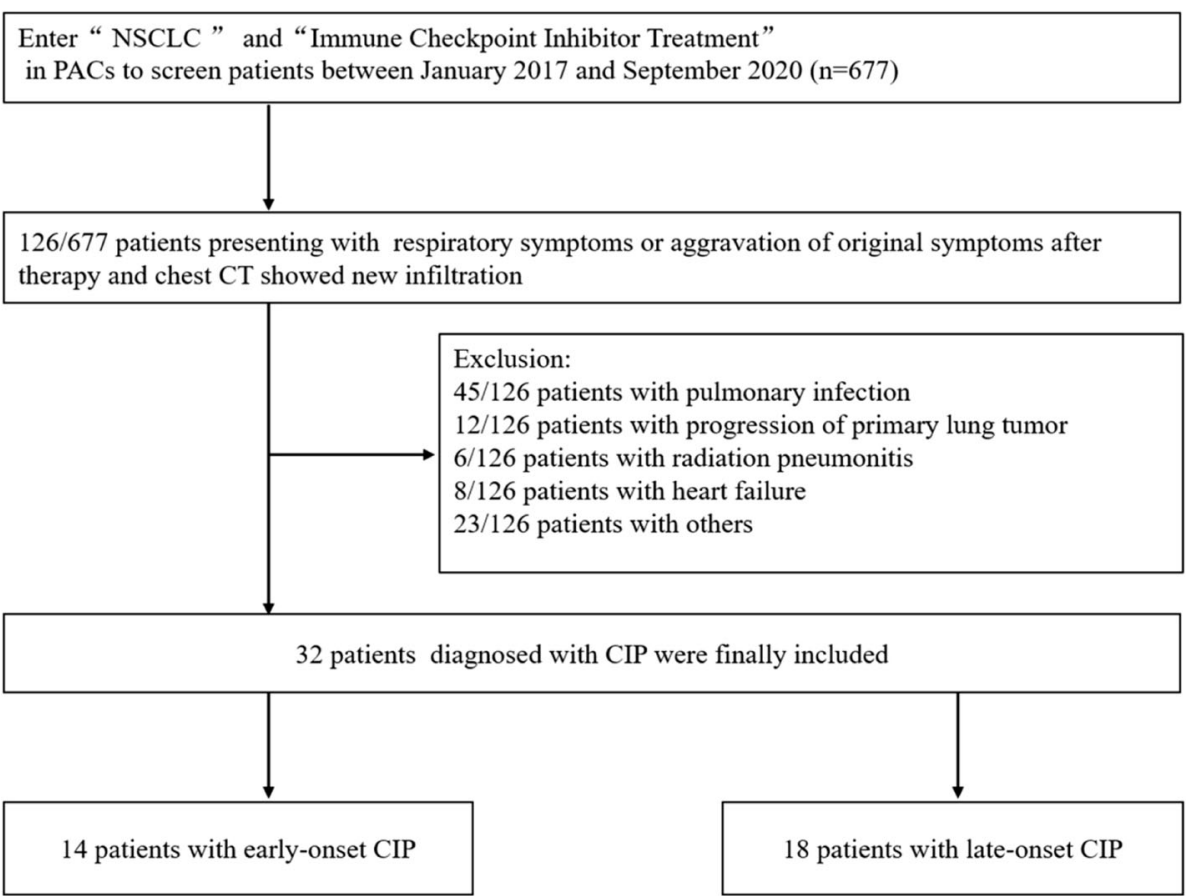

Fig. 1 Flowchart of Screening CIP Patients

requirement, increase in exercise capacity, or improvement in radiographic infiltrates after commencement of CIP treatment. Conversely, worsening was defined as lack of improvement in oxygen requirement and exercise capacity after $72 \mathrm{~h}$ of steroid therapy [16]. CIP that occurred within 6 weeks of ICI treatment and after 6 weeks of ICI treatment were considered early- and late-onset CIP, respectively [17]. Further, PTI was defined as ground glass opacity (GGO) confined to the area around the tumor [17]. CIP was graded using version 5.0 of the Common Terminology Criteria for Adverse Events (CTCAE, v5.0), published in November 2017 by the United States National Cancer Institute, as follows: grade 1: asymptomatic, clinical or diagnostic observations only, intervention not indicated; grade 2: symptomatic, medical intervention indicated, limiting instrumental activities of daily living (ADL); grade 3: severe symptoms, limiting self-care ADL, oxygen indicated; grade 4: liftthreatening respiratory compromise, urgent intervention indicated (e.g. tracheotomy or intubation); and grade 5: death [18].

\section{Chest CT}

In this study, all the patients underwent a non-contrast CT of the chest by the performance of a Siemens SOMATOM Sensation 64-Slice CT Scanner (Siemens, Forchheim, Germany) or a GE LightSpeed 16-Slice CT scanner (GE Healthcare, Beijing, China). CT related parameters were as follows: routine section thickness1.0,
1.25 , or $1.5 \mathrm{~mm}$; section thickness after reconstruction: $0.625-1.25 \mathrm{~mm}$; filtered back-projection reconstruction method; $80-120 \mathrm{kV} ; 200-280 \mathrm{mAs}$; and a B70f kernel. The data of these 677 patient's chest CT were procured from picture archiving and communication system (PACS).

\section{Chest CT interpretation}

The chest $\mathrm{CT}$ images were inspected by a thoracic radiologist (J.W., with 24 year-experience in cardiopulmonary imaging) and a medical student (Y.T., with 2 yearexperience in pulmonary imaging diagnosis) from each institution by using PACS (AGFA Healthcare, Mortsel, Belgium; lung window width, $1500 \mathrm{HU}$; level, $2500 \mathrm{HU}$ ) and traced the chest CT images that were highly suspected to be CIP in consensus. Whereafter, the presence of CIP in these patients were confirmed by a radiologist in pulmonary imaging (S.Z., with 17 year-experience in pulmonary imaging diagnosis) and a pulmonary radiologist (C.W., with 15 year-experience in pulmonary imaging diagnosis). Two radiologists mentioned above were analyzed the same patient independently on the same day, and the radiologists resolved the disagreement under discussion to reach a consensus. All radiologists were not aware of the pathologic diagnoses of the patients. The record of each lesion on the CT images included the number of involved lobes and lung area of CIP, consolidation, presence and distribution of GGO, nodularity, reticulation, pleural effusion, traction 
Table 1 Baseline Characteristics of 32 CIP Patients

\begin{tabular}{|c|c|}
\hline Characteristics & Datum \\
\hline Age, years, mean $\pm S D$ & $64.1 \pm 10.3$ \\
\hline Male, n (\%) & $26(81.3)$ \\
\hline Current smoking, n (\%) & $25(78.1)$ \\
\hline \multicolumn{2}{|l|}{ Symptoms, n (\%) } \\
\hline Cough & $17(53.1)$ \\
\hline Dyspnea & $29(90.6)$ \\
\hline Fever/Fatigue/Chest pain/No symptom & $11(11.3)$ \\
\hline \multicolumn{2}{|l|}{ Tumor location, n (\%) } \\
\hline Left lung & $13(40.6)$ \\
\hline Right lung & $19(59.4)$ \\
\hline \multicolumn{2}{|l|}{ Tumor histology, n (\%) } \\
\hline squamous & $9(28.1)$ \\
\hline adenocarcinoma & $20(59.4)$ \\
\hline Squamous+ adenocarcinoma & 2 \\
\hline others & $1(12.5)$ \\
\hline \multicolumn{2}{|l|}{ Initial tumor stage, $\mathrm{n}(\%)$} \\
\hline H-II & $1(3.1)$ \\
\hline III-IV & $31(96.9)$ \\
\hline \multicolumn{2}{|l|}{ ICl agents, n (\%) } \\
\hline pembrolizumab & $21(65.6)$ \\
\hline Nivolumab & $6(18.8)$ \\
\hline Sintilimab/durvalumab/nivolumab+ipilimumab & $5(15.6)$ \\
\hline Surgery, n (\%) & $7(21.9)$ \\
\hline Chemotherapy, n (\%) & $18(62.5)$ \\
\hline Radiotherapy, n (\%) & $8(25.0)$ \\
\hline Radiation pneumonitis, n (\%) & $2(6.3)$ \\
\hline \multicolumn{2}{|l|}{ Prognosis, n (\%) } \\
\hline Survival & $23(71.9)$ \\
\hline Death & $9(28.1)$ \\
\hline
\end{tabular}

bronchiectasis, and determination of the validity of ILD in new diffuse infiltration. Radiology severity of CIP stratified into mild, moderate, and severe [18]. CT findings of ILD were ranged according to the American Thoracic Society/European Respiratory Society (ATS/ ERS) international multidisciplinary classification of IP as AIP/DAD-like pattern, HP-like pattern, COP-like pattern, NSIP-like pattern, and others.

\section{Statistical methods}

Excel (Microsoft) was used for data collection, and SPSS version 26.0 (IBM Statistics, Armonk, NY, USA) was used for statistical analysis. Numerical data are expressed as mean \pm standard deviation. Chi-square or Fisher's exact test was used for categorical data. Patient survival was estimated by Kaplan-Meier method and compared with the log-rank test. $P<0.05$ was considered significant.

\section{Results}

\section{Clinical features}

Figure 1 shows the flowchart of screening the patients. A total of 32 (4.7\%) patients with CIP (mean age: $64.1 \pm$ 10.3 years; age range: $43-82$ years) were included in this study, including 26 (81.2\%) males (mean age: $64.0 \pm 10.3$ years; age range: $43-82$ years) and 6 (18.8\%) females (mean age: $64.5 \pm 11.3$ years; age range: $45-78$ years). The median onset time was 10 weeks (0.1-71 weeks), and early-onset and late-onset CIP accounted for 43.8 and $56.2 \%$, respectively. Among these patients, 25 (78.1\%) had a history of smoking, with an average of $40.4 \pm 31.5$ pack-years (range: $22.2-58.6$ pack-years). Of the 32 patients, $21(65.6 \%)$ patients were administered pembrolizumab, 6 (18.8\%) were administered nivolumab, $2(6.3 \%)$ were administered sintilimab, 2 (6.3\%) were administered durvalumab, and 1 (3.1\%) was administered nivolumab and ipilimumab. Thirty-two CIP patients received steroid treatment, after which, 23 (71.9\%) patients survived, including 17 patients' condition improved, 6 patients' condition worsened. In addition, 9 (28.1\%) patients died. Clinical features are detailed in Table 1.

\section{Radiographic patterns of CIP}

The chest CT patterns of the 32 patients with CIP included GGO (29 patients, 90.6\%), reticulation (14 patients, 43.8\%), consolidation (12 patients, 37.5\%), nodularity (8 patients, $25.0 \%)$, bronchitis (7 patients, $21.9 \%$ ), pleural effusion (2 patients; $6.3 \%$ ), and among which 5 (15.6\%) patients showed PTI features. The radiographic patterns of CIP included OP-like pattern (11 patients, 34.4\%; Fig. 2a), NSIP-like pattern (11 patients, 34.4\%; Fig. 2b), HP-like pattern (2 patients, 6.3\%; Fig. 2c), and AIP-like pattern (1 patient, 3.1\%; Fig. 2d).

\section{Difference in radiographic patterns of early-onset and late-onset CIP}

According to the occurrence time of CIP after ICI treatment, the patients were categorized into early-onset and late-onset CIP groups. In the early-onset CIP group, the chest CT patterns included GGO (13 patients, 92.9\%), consolidation (7 patients, 50.0\%), reticulation (5 patients, $35.7 \%$ ), nodularity (5 patients, $35.7 \%$ ), and bronchitis (3 patients, 21.4\%); whereas, in the late-onset CIP group, the chest CT patterns included GGO (16 patients, $88.9 \%$ ), reticulation (9 patients, $50.0 \%$ ), consolidation (5 patients, $27.8 \%$ ), nodularity (3 patients, $16.7 \%)$, bronchitis (4 patients, $22.2 \%$ ), and pleural effusion (2 patients, $11.1 \%)$, indicating that there was no statistical difference in the radiographic patterns between both the groups $(P>0.05)$.

The radiographic patterns of early-onset CIP included OP-like pattern (7 patients, 50.0\%), NSIP-like pattern (2 patients, 14.3\%), HP-like pattern (1 patient, 7.1\%), AIP- 

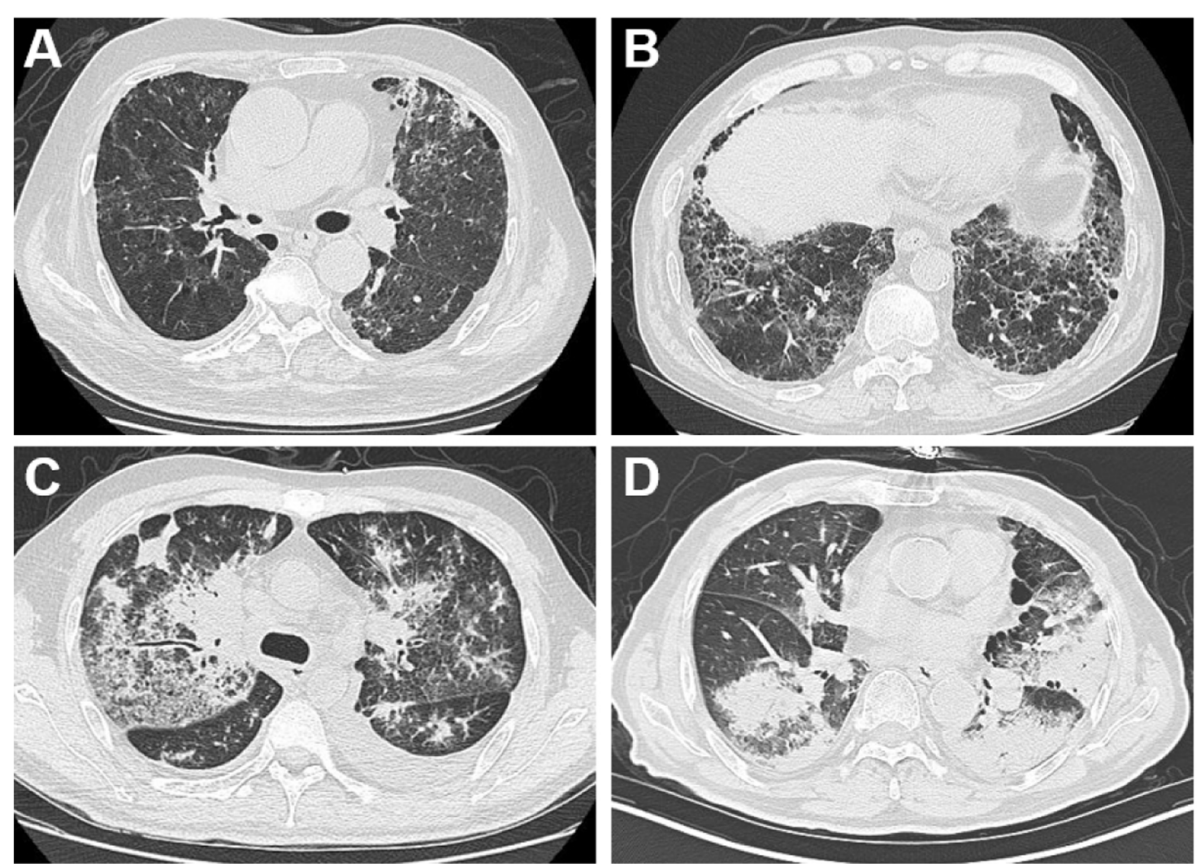

Fig. 2 Radiographic patterns of CIP. a HP-like pattern: GGO and nodules observed at left lung and right upper lung. b NSIP-like pattern: GGO and reticulation shadows observed at bilateral lungs. c AIP-like pattern: Diffuse GGO observed at bilateral upper lung and bilateral lower lobe dorsal segment, accompanied by consolidation and reticulation shadows. $\mathbf{d}$ OP-like pattern: Consolidation shadow and scattered GGO observed at left lung and right subpleural lower lung

like pattern (one patient, 7.1\%), and others (3 patients, $21.4 \%)$; whereas, the radiographic patterns of late-onset CIP included OP-like pattern (4 patients, 22.2\%), NSIPlike pattern ( 9 patients, 50.0\%), HP-like pattern (1 patient, 5.6\%), and others (4 patients, $22.2 \%$ ), thereby indicating that there was no statistical difference in the radiographic patterns between both the groups $(P>$ 0.05).

According to the grading system of CTCAE v5.0, there was one patient with grades 1-2 CIP (7.1\%) and 13 patients with grades 3-5 CIP (92.9\%) in the early-onset group. Whereas, in the late-onset group, 9 patients had grades 1-2 CIP (50.0\%) and 9 patients had grades 3-5 (50.0\%), statistical difference was observed in the CIP grading between both the groups $(P<0.05)$. In the earlyonset group, 7 patients (50.0\%) survived and 7 patients $(50.0 \%)$ died; whereas, in the late-onset group, 16 patients $(88.9 \%)$ survived and 2 patients (11.1\%) died, indicating a statistical difference in the overall survival (OS) between both the groups $(P<0.05$; Table 2 and Fig. 3$)$.

\section{Discussion}

ICIs have played an increasingly important role in the treatment of advanced malignant neoplasms in various systems [19]. However, with the expansion of clinical application, irAEs, especially fatal ones such as CIP, have drawn increasing attention. Sintilimab, a fully human anti-PD-1 monoclonal antibody, has a safety profile and effectiveness consistent with other approved PD-1 antibodies, which is only approved in China and used in the treatment of classic Hodgkin's lymphoma and NSCLC [20, 21]. Despite its infrequency, CIP shows no specificity in clinical findings but has complex radiographic patterns, which makes early diagnosis difficult. For some patients, a missed early diagnosis of CIP may have fatal consequences. Furthermore, CIP can occur at any time during the course of tumor immunotherapy. However, there is no report that determines the relationship between the occurrence time of CIP and prognosis. In this study, we found that there was a significant difference in the prognosis of early- and late-onset CIP patients. Accordingly, we proposed the concept of early-onset and late-onset CIP.

In multiple clinical trials, 3-7\% of patients had CIP after treatment with ICIs for solid tumors [22], among which approximately $0.8 \%$ were grade $\geq 3$ CIP [6]. In this study, we found that $4.7 \%$ of the patients had CIP, and grade $\geq 3$ CIP accounted for $68.8 \%$, which was significantly higher than the results of previous clinical trials. This difference may be associated with the patient selection in the mentioned clinical trials, where some patients with CIP high-risk factors (advanced age, complicating interstitial pneumonia, or radiation pneumonitis) were rejected. The median onset time of CIP is reportedly 2.8 months [18], and the median onset time in our study was 10 weeks (0.1-71 weeks), and early-onset and 
Table 2 Radiographic patterns of 14 early-onset CIPs and 18 late-onset CIPS

\begin{tabular}{|c|c|c|c|c|}
\hline & $\begin{array}{l}\text { Overall } \\
N=32\end{array}$ & $\begin{array}{l}\text { Early-onset CIP } \\
N=14\end{array}$ & $\begin{array}{l}\text { Late-onset CIP } \\
N=18\end{array}$ & $P$ \\
\hline \multicolumn{5}{|l|}{ CIP location, n (\%) } \\
\hline Bilateral & $25(78.1)$ & $10(71.4)$ & $15(83.3)$ & \multirow[t]{3}{*}{$0.459^{*}$} \\
\hline Left & $4(12.5)$ & $3(21.4)$ & $1(5.6)$ & \\
\hline Right & $3(9.4)$ & $1(7.2)$ & $2(11.1)$ & \\
\hline \multicolumn{5}{|c|}{ Number of lobes involved, n (\%) } \\
\hline $1-3$ & $10(31.3)$ & $5(35.7)$ & $7(38.9)$ & \multirow[t]{2}{*}{$0.712^{*}$} \\
\hline $4-5$ & $22(68.7)$ & $9(64.3)$ & $11(61.1)$ & \\
\hline \multicolumn{5}{|c|}{ Involves area of lung parenchyma } \\
\hline$\leq 50 \%$ & $14(43.8)$ & $4(28.6)$ & $11(61.1)$ & \multirow[t]{2}{*}{$0.087^{*}$} \\
\hline$>50 \%$ & $18(56.2)$ & $10(71.4)$ & $7(38.9)$ & \\
\hline \multicolumn{5}{|c|}{$\mathrm{CT}$ findings at onset of CIP, $\mathrm{n}(\%)$} \\
\hline Ground glass opacity & $29(90.6)$ & $13(92.9)$ & $16(88.9)$ & \multirow[t]{5}{*}{$0.598^{*}$} \\
\hline Consolidation & $12(37.5)$ & $7(50.0)$ & $5(27.8)$ & \\
\hline Reticulation & $14(43.8)$ & $5(35.7)$ & $9(50.0)$ & \\
\hline Bronchitis/Nodularity & $15(46.9)$ & $8(57.1)$ & $7(38.9)$ & \\
\hline Pleural effusion & $2(6.3)$ & $0(0)$ & $2(11.1)$ & \\
\hline \multicolumn{5}{|l|}{ Overall pattern of ILD, n (\%) } \\
\hline OP-like pattern & $11(34.4)$ & $7(50.0)$ & $4(22.2)$ & \multirow[t]{3}{*}{$0.096^{*}$} \\
\hline NSIP-like pattern & $11(34.4)$ & $2(14.3)$ & $9(50.0)$ & \\
\hline AIP/HP/Others-like pattern & $10(31.3)$ & $5(35.7)$ & $5(27.8)$ & \\
\hline \multicolumn{5}{|l|}{ Grades of CIP, n (\%) } \\
\hline $1-2$ & $10(31.3)$ & $1(7.1)$ & $9(50.0)$ & \multirow[t]{2}{*}{$0.019^{*}$} \\
\hline $3-5$ & $22(68.7)$ & $13(92.9)$ & $9(50.0)$ & \\
\hline \multicolumn{5}{|l|}{ Prognosis, n (\%) } \\
\hline Survival & $23(71.9)$ & $7(50.0)$ & $16(88.9)$ & \multirow[t]{2}{*}{$0.022^{*}$} \\
\hline Dead & $9(28.1)$ & $7(50.0)$ & $2(11.1)$ & \\
\hline
\end{tabular}

* Fisher exact test

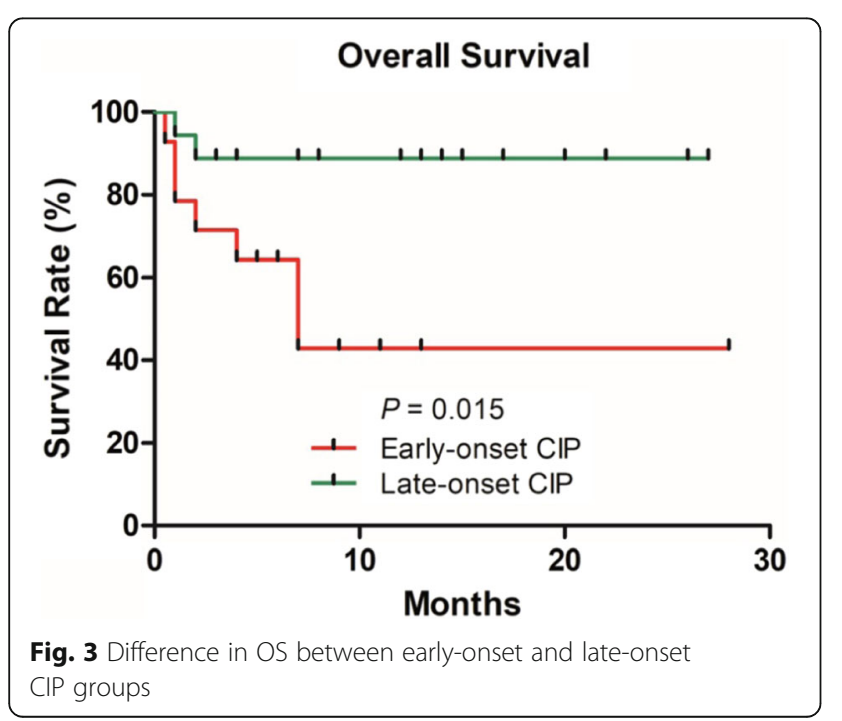

late-onset CIP accounted for 43.8 and 56.2\%, respectively. In early-onset CIP, $59.1 \%$ were grade $\geq 3$ CIP, indicating that severe patients often had CIP in the early stage of ICI treatment despite the low incidence of CIP. Baba et al. [23] found that the mortality rate of nivolumab-related interstitial pulmonary diseases was $17.4 \%$. In this study, the mortality rate of CIP was $28.1 \%$. After group assignment, it was found that the mortality of the early-onset CIP group (50.0\%) was significantly higher than that of the late-onset CIP group (11.1\%). The results of the log-rank test suggested that the median survival time of the earlyonset CIP group was only 7 months, and the OS rate of the early-onset CIP group was significantly lower than that of the late-onset CIP group. Thus, physicians should identify high-risk patients during the early stages and perform regular follow-ups to ensure an early diagnosis and treatment. 
A
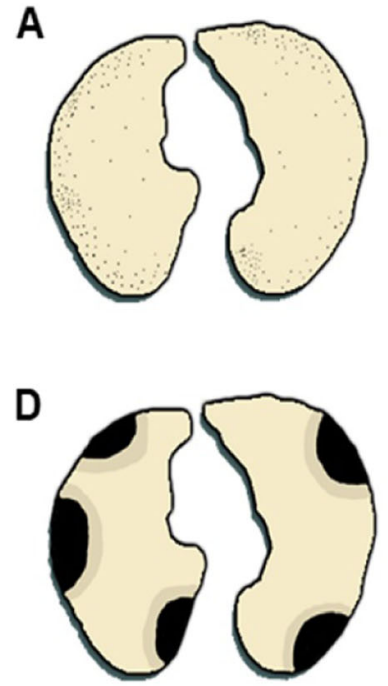

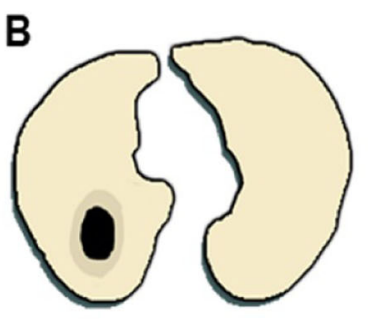

C

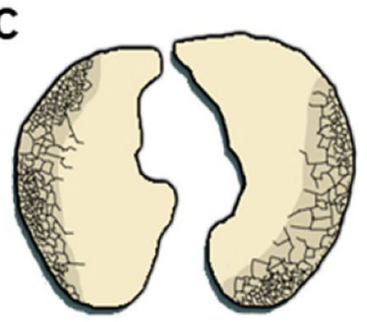

$\mathrm{E}$
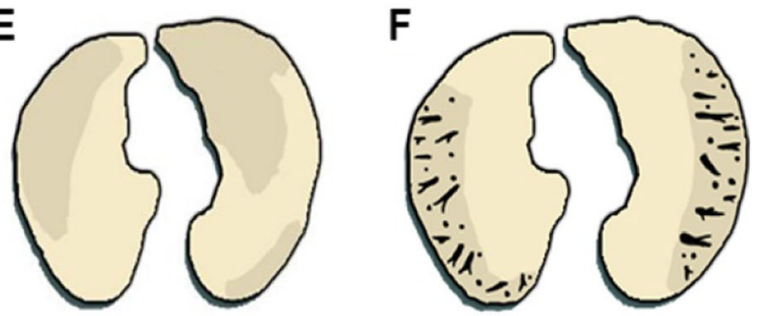

Fig. 4 Chest CT patterns of CIP. a Nodularity. b PTI. c Reticulation. d Consolidation. e Ground glass opacity. f Bronchitis

CIP has various radiographic patterns characterized by nodularity, PTI, reticulation, consolidation, GGO, interlobular septal thickening, and funicular opacity (Fig. 4). According to the ATS/ERS international multidisciplinary classification of IP [24], the radiographic patterns of CIP are characterized by NSIP-, OP-, GGO-, and AIP- like patterns. This study found that CIP was often involved in multiple lobes and segments of both lungs, and radiographic patterns were consistent with those reported in the literature, where the most common pattern was GGO, followed by OP-like, NSIP-like, bronchitislike, nodularity, and AIP-like patterns. Meghan Shea

Table 3 Radiologic severity of 32 CIP Patients

\begin{tabular}{|c|c|c|c|}
\hline Severity & Mild & Moderate & Severe \\
\hline CT Image & 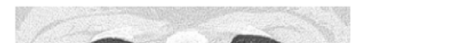 & & \\
\hline Description & $\begin{array}{l}\text { Confined to one lobe of the lung or } \\
\text { Confined to }<25 \% \text { of lung parenchyma }\end{array}$ & $\begin{array}{l}\text { Involves more than one lobe of the lung or } \\
\text { Involves } 25-50 \% \text { of lung parenchyma }\end{array}$ & $\begin{array}{l}\text { Involves all lobes of the lung or } \\
\text { Involves }>50 \% \text { of lung parenchyma }\end{array}$ \\
\hline $\begin{array}{l}\text { Early-onset } \\
\text { CIP }\end{array}$ & $\mathrm{n}$ & $\mathrm{n}$ & $n$ \\
\hline $\begin{array}{l}\text { COP } \\
\text { pattern }\end{array}$ & 1 & 1 & 5 \\
\hline $\begin{array}{l}\text { NSIP } \\
\text { pattern }\end{array}$ & 0 & 0 & 2 \\
\hline $\begin{array}{l}\text { Other } \\
\text { patterns }\end{array}$ & 0 & 2 & 3 \\
\hline \multicolumn{4}{|l|}{$\begin{array}{l}\text { Late-onset } \\
\text { CIP }\end{array}$} \\
\hline $\begin{array}{l}\text { COP } \\
\text { pattern }\end{array}$ & 0 & 3 & 1 \\
\hline $\begin{array}{l}\text { NSIP } \\
\text { pattern }\end{array}$ & 0 & 3 & 6 \\
\hline $\begin{array}{l}\text { Other } \\
\text { patterns }\end{array}$ & 0 & 2 & 3 \\
\hline
\end{tabular}


et al. reported that one patient had fatal CIP after treatment with pembrolizumab, and the post-onset chest $\mathrm{CT}$ suggested an OP-like change [25]. Nishino et al. reported that two patients receiving nivolumab treatment for advanced NSCLC had CIP in the early stages after treatment, and their chest CT suggested GGO and OPlike changes [10]. Nishino et al. also reported the chest CT findings and radiographic patterns of 20 patients with malignant tumors (10 with malignant melanoma, 6 with lymphoma, and 4 with pulmonary malignancy) who had developed CIP after treatment with immune checkpoint blockade [26]. Out of 20 patients, 7 had developed CIP in early stages (CIP onset time since treatment: 0.51.4 months). Their chest $\mathrm{CT}$ were mainly characterized by GGO, reticular opacity, and consolidation; whereas, 6 patients $(85.7 \%)$ showed an OP-like pattern, 1 patient (14.3\%) showed an HP-like pattern, and no patients presented with an NSIP-like pattern. Thirteen patients developed CIP in the late stage (CIP onset time since treatment: $1.6-11.5$ months). Moreover, in addition to GGO, reticular opacity, and consolidation patterns, bronchodilation and centrilobular nodularity were also observed. In terms of radiographic patterns, 7 patients $(53.8 \%)$ had an OP-like pattern, 3 (23.1\%) patients had an NSIP-like pattern, two (15.4\%) patients had an AIP-like pattern, and one (7.7\%) patient had an HPlike pattern. Therefore, the main radiographic patterns in early-onset CIP was an OP-like pattern, while the NSIP-like pattern was seen more frequently in lateonset CIP patients. The results of this study were consistent with those reported in the literature, where we identified 7 (50.0\%) patients with an OP-like pattern and $3(21.4 \%)$ patients with NSIP-like pattern in the early-onset group, compared to 9 (50.0\%) patients with an NSIP-like pattern and five (27.8\%) patients with an OP-like pattern in the late-onset group.

What was the reason for the difference in prognosis between early-onset CIP and late-onset CIP? Firstly, there were more grade $\geq 3 \mathrm{CIP}$ patients in early-onset CIP; secondly, patients with early-onset CIP had higher radiologic severity grade $(71.4 \% \mathrm{v}$ $55.6 \%)$. It is generally believed that the prognosis of COP pattern is better. But in this study, the mortality of early-onset CIP with COP pattern as the main manifestation was higher, which was related to the higher proportion of severe radiologic severity in early-onset CIP group (71.4\% v 25.0\%) (Table 3).

\section{Conclusion}

Our cohort provides a new insight into the difference of radiographic pattern and prognosis between earlyonset and late-onset CIP. We have shown that earlyonset CIP patients often have more severe symptoms and poorer prognosis, with an OP-like pattern as the dominant radiographic pattern. Late-onset CIP patients often have fewer symptoms and better prognosis, with NSIP-like pattern as the dominant radiographic pattern. When CIP develops in clinical settings, attention should be paid to the onset time, grading, and radiographic pattern of CIP; consequently, immediate diagnosis must be performed and treatment should be provided to improve the prognosis. The current study has several limitations, including its retrospective design, small sample size, and lack of histopathological and bronchoscopic findings. Thus, additional studies with larger sample sizes are required to confirm our results.

\section{Abbreviations \\ ICl: Immune checkpoint inhibitors; NSCLC: Non-small cell lung cancer; CIP: Checkpoint inhibitor-associated pneumonia; irAE: Immune-related adverse event; CT: Computed tomography; COP: Cryptogenic organizing pneumonia; NSIP: Nonspecific interstitial pneumonia; AIP: Acute interstitial pneumonia; EGFR-TKI: Epidermal growth factor receptor-tyrosine kinase in- hibitor; PTI: Peritumoral Infiltration; GGO: Ground glass opacity; NCCN: National Comprehensive Cancer Network; PACS: Picture archiving and communication system; ATS/ERS: American Thoracic Society/European Respiratory Society}

\section{Supplementary Information}

The online version contains supplementary material available at https://doi. org/10.1186/s12885-021-08353-y.

\section{Additional file 1.}

\section{Acknowledgements}

This work was supported by the Excellent talents in Beijing "Youth top team" (No. 2019YXBJ2), National Natural Science Foundation of China (No. 81902324) and Beijing Hospitals Authority Clinical Medicine Development of special funding support.

\section{Authors' contributions}

A.H., Y.X., S.L., G.Z., and X.X. conceptualized the article. A.H., Y.X., X.Z., C.W. J.G., X.L., M.X., X.M., H.D., J.S., F.R., L.P., J.Q., Z.Y., S.W., Y.C., L.P., and X.X. analyzed the data. A.H., Y.X., X.Z., and X.X. wrote the initial draft. G.Z., S.L., and $X . X$. are the guarantors. The author(s) read and approved the final manuscript.

\section{Funding}

National Natural Science Fund Youth Project (81700007); National Natural Science Foundation of China (81772798, 62041602); Research and innovation fund of the Ministry of Education (2018A03026); Beijing Natural Science Foundation (2019A10); "Qingmiao" plan of Beijing Municipal Hospital Administration (2018QM4); Outstanding top talent (2019YXBJ1); Capital health development scientific research unit matching fund (2020-2Z2086). National Multidisciplinary Cooperative Diagnosis and Treatment Capacity Project for Major Diseases (2019YLFW); Beijing Clinical Key Specialty Project (2020ZDZK1).

Availability of data and materials

All the data generated and analyzed during this study are included in this published article.

\section{Declarations}

Ethics approval and consent to participate

This study was approved by the Clinical Trial Ethics Committee of Beijing Shijitan Hospital (sjtky11-1x-2020 (74)) and informed consent forms were obtained from all patients prior to their participation in this study. All 
methods were carried out in accordance with relevant guidelines and regulations.

\section{Consent for publication}

Not applicable.

\section{Competing interests}

The authors declare that the research was conducted in the absence of any commercial or financial relationships that could be construed as a potential conflict of interest.

\section{Author details}

'Department of Respiratory and Critical Care, Beijing Shijitan Hospital, Capital Medical University, Beijing, China. ${ }^{2}$ Department of Respiratory and Critical Care, Chinese PLA General Hospital, Beijing, China. ${ }^{3}$ Center of Clinical Laboratory Medicine, the first Medical Centre, Chinese PLA General Hospital, Beijing, China. ${ }^{4}$ Department of Radiology, Chinese PLA General Hospital, Beijing, China. ${ }^{5}$ Department of Pathology, Chinese PLA General Hospital, Beijing, China. ${ }^{6}$ Department of Radiology, Affiliated Zhongshan Hospital of Dalian University, Dalian, China. ${ }^{7}$ Department of Respiratory Medicine, Weifang Medical University, Weifang, China. ${ }^{8}$ School of Medicine, Peking University, Beijing, China. ${ }^{9}$ Shanghai Key Laboratory of Gynecologic Oncology, Renji Hospital, School of Medicine, Shanghai Jiao Tong University, Shanghai, China. ${ }^{10}$ Institute of Interdisciplinary Integrative Medicine Research, Shanghai University of Traditional Chinese Medicine, Shanghai, China.

Received: 8 February 2021 Accepted: 11 May 2021

Published online: 29 May 2021

\section{References}

1. Reardon DA, Brandes AA, Omuro A, Mulholland P, Lim M, Wick A, et al. Effect of Nivolumab vs bevacizumab in patients with recurrent glioblastoma: the CheckMate 143 phase 3 randomized clinical trial. JAMA Oncol. 2020;6(7):1003-10.

2. Madden K, Kasler MK. Immune checkpoint inhibitors in lung cancer and melanoma. Semin Oncol Nurs. 2019;35(5):150932

3. Kennedy LB, Salama AKS. A review of cancer immunotherapy toxicity. CA Cancer J Clin. 2020;70(2):86-104.

4. Hsiehchen D, Watters MK, Lu R, Xie Y, Gerber DE. Variation in the assessment of immune-related adverse event occurrence, grade, and timing in patients receiving immune checkpoint inhibitors. JAMA Netw Open. 2019;2(9):e1911519.

5. Pozzessere C, Bouchaab H, Jumeau R, Letovanec I, Daccord C, Bourhis J, et al. Relationship between pneumonitis induced by immune checkpoint inhibitors and the underlying parenchymal status: a retrospective study. ERJ Open Res. 2020;6(1):00165-2019.

6. Nishino M, Giobbie-Hurder A, Hatabu H, Ramaiya NH, Hodi FS. Incidence of programmed cell death 1 inhibitor-related pneumonitis in patients with advanced cancer: a systematic review and meta-analysis. JAMA Oncol. 2016; 2(12):1607-16

7. Gettinger SN, Horn L, Gandhi L, Spigel DR, Antonia SJ, Rizvi NA, et al. Overall survival and long-term safety of nivolumab (anti-programmed death 1 antibody, BMS-936558, ONO-4538) in patients with previously treated advanced non-small-cell lung cancer. J Clin Oncol. 2015;33(18):2004-12.

8. Garon EB, Rizvi NA, Hui R, Leighl N, Balmanoukian AS, Eder JP, et al. Pembrolizumab for the treatment of non-small-cell lung cancer. N Engl J Med. 2015;372(21):2018-28.

9. Cadranel J, Canellas A, Matton L, Darrason M, Parrot A, Naccache JM, et al. Pulmonary complications of immune checkpoint inhibitors in patients with nonsmall cell lung cancer. Eur Respir Rev. 2019;28(153):190058.

10. Chuzi S, Tavora F, Cruz M, Costa R, Chae YK, Carneiro BA, et al. Clinical features, diagnostic challenges, and management strategies in checkpoint inhibitor-related pneumonitis. Cancer Manag Res. 2017;9:207-13.

11. Nakahama K, Tamiya A, Taniguchi Y, Sasaki Y, Akira M, Atagi S. Severe acute interstitial lung disease after nivolumab in three non-small cell lung cancer patients with imaging findings of airway obstruction adjacent to lung tumors. J Infect Chemother. 2017;23(12):826-9.

12. Tonk EHJ, van Lindert ASR, Verhoeff JJC, Suijkerbuijk KPM. Acute-onset pneumonitis while administering the first dose of durvalumab. Case Rep Oncol. 2019;12(2):621-4.
13. Costa RB, Benson A, Yaghmai V, Costa RLB, Zhou H, Behdad A, et al. An extremely rapid case of pneumonitis with the use of nivolumab for pancreatic adenocarcinoma. Case Rep Oncol Med. 2018;2018:6314392.

14. Nishino M, Sholl LM, Awad MM, Hatabu H, Armand P, Hodi FS. Sarcoid-like granulomatosis of the lung related to immune-checkpoint inhibitors: distinct clinical and imaging features of a unique immune-related adverse event. Cancer Immunol Res. 2018;6(6):630-5.

15. Suresh K, Voong KR, Shankar B, Forde PM, Ettinger DS, Marrone KA, et al. Pneumonitis in non-small cell lung cancer patients receiving immune checkpoint immunotherapy: incidence and risk factors. J Thorac Oncol. 2018;13(12):1930-9.

16. Suresh K, Naidoo J, Lin CT, Danoff S. Immune checkpoint immunotherapy for non-small cell lung cancer: benefits and pulmonary toxicities. Chest. 2018;154(6):1416-23.

17. Teraoka S, Fujimoto D, Morimoto T, Kawachi H, Ito M, Sato Y, et al. Early immune-related adverse events and association with outcome in advanced non-small cell lung cancer patients treated with nivolumab: a prospective cohort study. J Thorac Oncol. 2017;12(12):1798-805.

18. Naidoo J, Wang X, Woo KM, lyriboz T, Halpenny D, Cunningham J, et al. Pneumonitis in patients treated with anti-programmed death-1/ programmed death ligand 1 therapy. J Clin Oncol. 2017;35(7):709-17.

19. Dizon DS, Krilov L, Cohen E, Gangadhar T, Ganz PA, Hensing TA, et al. Clinical cancer advances 2016: annual report on progress against cancer from the American Society of Clinical Oncology. J Clin Oncol. 2016;34(9): 987-1011.

20. Gao S, Li N, Gao S, Xue Q, Ying J, Wang S, et al. Neoadjuvant PD-1 inhibitor (Sintilimab) in NSCLC. J Thorac Oncol. 2020;15(5):816-26.

21. Shi $Y$, Su H, Song $Y$, Jiang $W$, Sun $X$, Qian $W$, et al. Safety and activity of sintilimab in patients with relapsed or refractory classical Hodgkin lymphoma (ORIENT-1): a multicentre, single-arm, phase 2 trial. Lancet Haematol. 2019;6(1):e12-9.

22. Khunger M, Rakshit S, Pasupuleti V, Hernandez AV, Mazzone P, Stevenson J, et al. Incidence of pneumonitis with use of programmed death 1 and programmed death-ligand 1 inhibitors in non-small cell lung cancer: a systematic review and meta-analysis of trials. Chest. 2017;152(2):271-81.

23. Baba T, Sakai F, Kato T, Kusumoto M, Kenmotsu H, Sugiura H, et al. Radiologic features of pneumonitis associated with nivolumab in non-smallcell lung cancer and malignant melanoma. Future Oncol. 2019;15(16):191120.

24. Travis WD, Costabel U, Hansell DM, King TE Jr, Lynch DA, Nicholson AG, et al. An official American Thoracic Society/European Respiratory Society statement: update of the international multidisciplinary classification of the idiopathic interstitial pneumonias. Am J Respir Crit Care Med. 2013;188(6): 733-48.

25. Shea M, Rangachari D, Hallowell RW, Hollie NI, Costa DB, VanderLaan PA. Radiologic and autopsy findings in a case of fatal immune checkpoint inhibitor-associated pneumonitis. Cancer Treat Res Commun. 2018;15:17-20.

26. Nishino M, Ramaiya NH, Awad MM, Sholl LM, Maattala JA, Taibi M, et al. PD1 inhibitor-related pneumonitis in advanced cancer patients: radiographic patterns and clinical course. Clin Cancer Res. 2016;22(24):6051-60.

\section{Publisher's Note}

Springer Nature remains neutral with regard to jurisdictional claims in published maps and institutional affiliations.

Ready to submit your research? Choose BMC and benefit from:

- fast, convenient online submission

- thorough peer review by experienced researchers in your field

- rapid publication on acceptance

- support for research data, including large and complex data types

- gold Open Access which fosters wider collaboration and increased citations

- maximum visibility for your research: over $100 \mathrm{M}$ website views per year

At BMC, research is always in progress.

Learn more biomedcentral.com/submissions 